\title{
Intrinsic Neurons and Nonvascular Smooth Muscle Cells in the Choroid of a Chimpanzee (Pan troglodytes)
}

\author{
Christian Albrecht May ${ }^{1}$ (D)
}

Received: 28 August 2017 / Accepted: 5 February 2018 / Published online: 13 March 2018 (C) Springer Science+Business Media, LLC, part of Springer Nature 2018

Visual adjustment is a key skill in primate development and has received a great deal of attention in recent years. The evolution of trichromatic vision in some primates (Wells et al. 2008; Kawamura 2016) facilitates behaviors such as mate choice (Fernandez and Morris 2007; Fornalé et al. 2012) and the identification of nourishing food resources (Dominy and Lucas 2001; Jacobs and Bradley 2016; Melin et al. 2013). The evolution of binocular vision is relevant to visual attention and improves stereoscopic perception (Heesy 2009). The specialization of visual pathways in the brain led to similar face scanning in humans and apes, with the exception of human-specific prolonged eye viewing (Kano et al. 2012). Fine-tuned vision is maintained by the whole ocular apparatus, including a specialized uveal tract consisting of the iris, the ciliary body, and the choroid.

The choroid has several functions, including nutrition of the outer and parts of the inner retina, thermoregulation, adjustment of the eye's shape, and adjustment of the fovea centralis for precise vision (Nickla and Wallman 2010). The latter is thought to be particularly developed in humans. Morphological structures attributed to this function are a high number, 1000-2500, of intrinsic choroidal neurons (May and Lütjen-Drecoll 2005) and a complex system of nonvascular smooth muscle cells (May 2005). In contrast, primates with a sparsely developed foveal system have low numbers of neurons (Aotes trivirgatus, Tupaia glis, Macaca fascicularis, Macaca mulatta: Flügel-Koch et al. 1994) and a less developed nonvascular smooth muscle cell system (Aotes trivirgatus, Tupaia glis, Cercocebus, Macaca fascicularis, Macaca mulatta:

Handling Editor: Joanna M. Setchell.

Christian Albrecht May

Albrecht.May@tu-dresden.de

1 Department of Anatomy, Medical Faculty Carl Gustav Carus, TU Dresden, 01307 Dresden, Germany 

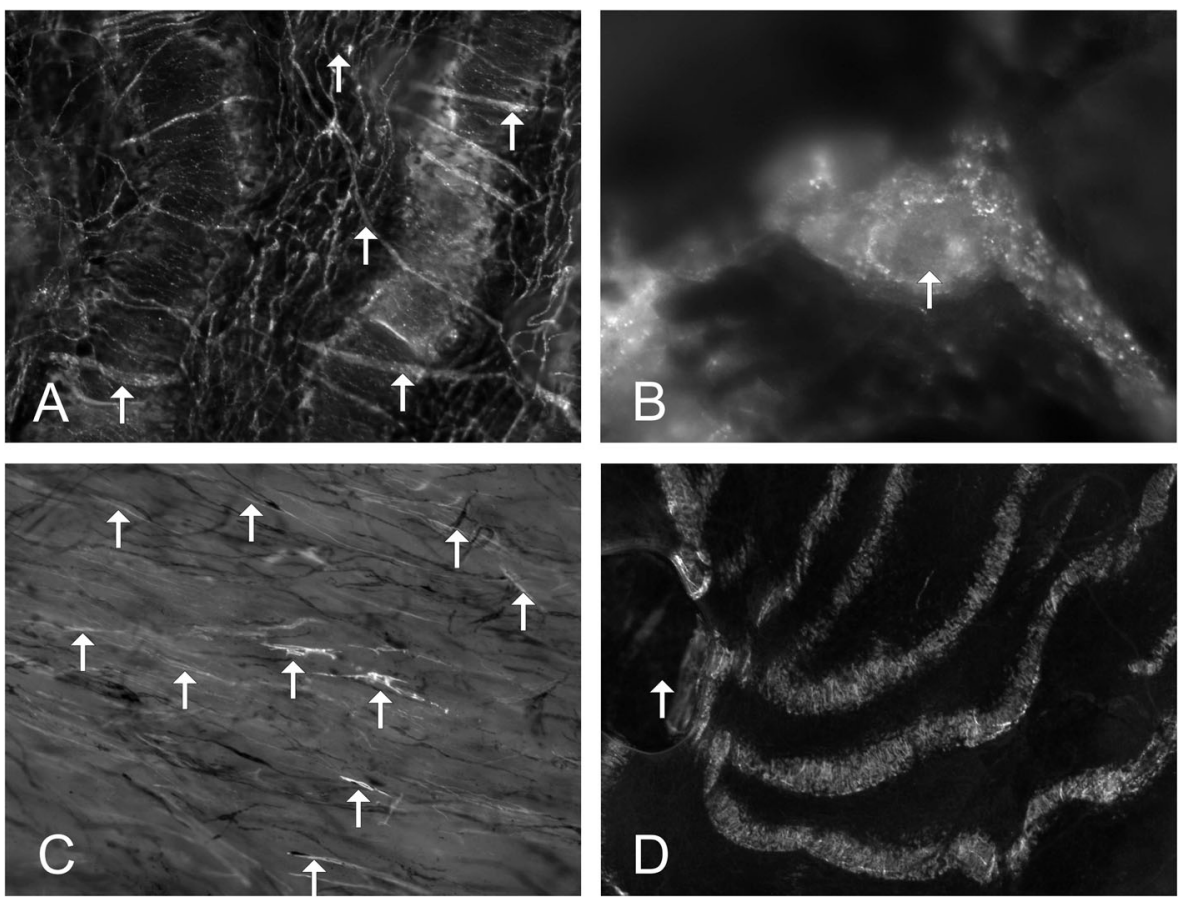

Fig. 1 Choroidal and scleral whole mounts of the posterior of an eye of Pan troglodytes, stained for protein gene product 9.5 (a, b) and smooth muscle $\alpha$-actin (c, d). Arrows in a show bundles of the suprachoroidal nerve plexus crossing the vessels obliquely next to a dense perivascular nerve plexus running parallel with the vessels. Arrows in b show intrinsic neurons in the suprachoroidal nerve plexus. Arrows in c show a few nonvascular smooth muscle cells in the suprachoroidal layer oriented parallel to the pigmented cell processes of the inner sclera. $\mathbf{d}$ shows that only vascular smooth muscle cells were seen in the choroid. The arrow in $\mathbf{d}$ marks the region of a vortex vein

May 2003). Only the general anatomy of the choroid in apes is currently known. To understand the importance of a fine-tuning choroid for specialized visual functions, we need to determine whether the fine choroidal morphology of apes is more similar in structure and function to that of humans or of monkeys. In this study, I aimed to investigate the intrinsic neurons and nonvascular smooth muscle cells of a Pan troglodytes, using whole mount preparations.

I received a pair of eyes of Pan troglodytes from Zagreb Zoo, where a female Pan troglodytes born 1985 in Hannover, Germany (no subspecies provided), died at the age of 31 years. In Zagreb, the eyes were enucleated 3 days postmortem and fixed in formalin.

I made whole mounts of the complete sclera and choroid of one eye and bleached the choroid with $1 \% \mathrm{H}_{2} \mathrm{O}_{2}$ for $4 \mathrm{~h}$. I rinsed the whole mounts in phosphate-buffered saline (PBS, pH 7.4) and incubated them with the pan-neuronal marker protein gene product 9.5 (rabbit anti-PGP 9.5; dilution 1:400) and smooth muscle specific actin (mouse anti- $\alpha \mathrm{SMA}$; dilution 1:150) overnight at $6{ }^{\circ} \mathrm{C}$. The incubation solution also contained $10 \%$ Triton X and $1 \%$ goat serum solution in PBS. After washing the whole mounts 3 times in PBS for $10 \mathrm{~min}$ each, I incubated them with an appropriate 
secondary fluorescent antibody (goat anti-rabbit Cy3; dilution 1:500 and goat antimouse FITC; dilution 1:200) for $2 \mathrm{~h}$ at room temperature. After washing the whole mounts in PBS again, I mounted them with glycerin jelly and viewed them under a fluorescence microscope (Olympus BX 60 with an F-View II CCD camera).

I made sections of the four quadrants of the other eye (temporal, nasal, superior, and inferior) and embedded them in paraffin. I stained sagittal sections with hematoxylineosin and examined them with a light microscope (Leica DM1000).

My light microscopic observations of the choroidal sections showed well developed choroidal layers and no signs of pathology. I measured the thickness of the choroid in different regions and found it to be $426 \mu \mathrm{m}$ in the fovea, 200-220 $\mu \mathrm{m}$ in the peripapillary region, and $100-130 \mu \mathrm{m}$ at the ora serrata.

The whole mounts showed an elaborate network of PGP 9.5-positive nerve fibers around the blood vessels and in the suprachoroid (Fig. 1a). I detected 260 intrinsic neurons in the suprachoroidal plexus but these did not stain brightly for PGP 9.5 (Fig. 1b). They often formed groups of up to 15 cells.

I detected smooth muscle actin in all vessel walls. In contrast, nonvascular smooth muscle cells were sparsely located in the suprachoroidal layer, predominantly in the central temporal region. The few cells were oriented from the optic nerve toward the periphery (Fig. 1c). I did not find any clusters around the entrance of the ciliary arteries and nerves or near the vortex veins (Fig. 1d).

The general morphology of the choroidal vessels of Pan troglodytes and choroidal thickness are comparable to those described for Gorilla gorilla gorilla (Knapp et al. 2007) and humans (Hogan et al. 1971). In contrast, specific morphological structures attributed to fine regulation of the human choroid were only sparsely present in the pair of eyes of Pan troglodytes I studied. The number and arrangement of nonvascular smooth muscle cells in the choroid of Pan troglodytes was less developed than described for Macaca fascicularis and Macaca mulatta (May 2003). The number of the intrinsic neurons was lower than in humans (May and Lütjen-Drecoll 2005) but comparable to that in Macaca mulatta (range 326-601 intrinsic neurons; May et al. 2006). There are no reported or observed pathologies that suggest that this specific Pan troglodytes is atypical. Based on the findings, I hypothesize that the elaborate fine-tuning ability of the choroid is the last morphological step in the evolution of the human eye.

Further studies are needed to confirm and build on these findings and to understand eye morphology and physiology further. For example, in vivo ultrasonic investigations could compare the fine-tuning mechanism of the choroid between human and apes to test the function of the intrinsic choroidal neurons and the nonvascular smooth muscle cells. In addition, studies could investigate the morphological correlates of specific visual abilities such as face scanning (Kano et al. 2012) or binocular coordination (Heesy 2009).

Acknowledgements I thank Dijana Beneta (Zoo Zagreb) for providing the eyes investigated, and the editorin-chief Joanna Setchell and reviewers for fruitful comments and extensive effort in shaping the manuscript and improving its quality. There was no funding of this research.

\section{Compliance with Ethical Standards}

Conflicts of Interest The author has no conflicts of interest. 


\section{References}

Dominy, N. J., \& Lucas, P. W. (2001). Ecological importance of trichromatic vision to primates. Nature, 410, 363-366.

Fernandez, A. A., \& Morris, M. R. (2007). Sexual selection and trichromatic color vision in primates: Statistical support for the preexisting-bias hypothesis. The American Naturalist, 170, 10-20.

Flügel-Koch, C., Kaufman, P., \& Lütjen-Drecoll, E. (1994). Association of a choroidal ganglion cell plexus with the fovea centralis. Investigative Ophthalmology \& Visual Science, 35, 4268-4272.

Fornalé, F., Vaglio, S., Spiezio, C., \& Previde, E. P. (2012). Red-green color vision in three catarrhine primates. Communicative \& Integrative Biology, 5, 583-589.

Heesy, C. P. (2009). Seeing in stereo: The ecology and evolution of primate binocular vision and stereopsis. Evolutionary Anthropology, 18, 21-35.

Hogan, M. J., Alvarado, J. A., \& Weddell, J. E. (1971). Histology of the human eye. Philadelphia: W. B. Saunders.

Jacobs, R. L., \& Bradley, B. J. (2016). Considering the influence of nonadaptive evolution on primate color vision. PLoS One, 11, e0149664.

Kano, F., Call, J., \& Tomonaga, M. (2012). Face and eye scanning in gorillas (Gorilla gorilla), orangutans (Pongo abelii), and humans (Homo sapiens): Unique eye-viewing patterns in humans among hominids. Journal of Comparative Psychology, 126, 388-398.

Kawamura, S. (2016). Color vision diversity and significance in primates inferred from genetic and field studies. Genes \& Genomics, 38, 779-791.

Knapp, S., McCulley, J. P., Alvarado, T. P., \& Hogan, R. N. (2007). Comparative ocular anatomy of the western lowland gorilla. Veterinary Ophthalmology, 10, 357-362.

May, C. A. (2003). Nonvascular smooth muscle $\alpha$-actin positive cells in the choroid of higher primates. Current Eye Research, 27, 1-6.

May, C. A. (2005). Nonvascular smooth muscle cells in the human choroid: Distribution, development and further characterization. Journal of Anatomy, 207, 381-390.

May, C. A., Kaufman, P. L., Lütjen-Drecoll, E., \& Scholz, M. (2006). Choroidal innervation and optic neuropathy in macacque monkeys with laser- or anterior chamber perfusion-induced short-term elevation of intraocular pressure. Experimental Eye Research, 82, 1060-1067.

May, C. A., \& Lütjen-Drecoll, E. (2005). Choroidal ganglion cells in prenatal, young and middle-aged human donor eyes. Current Eye Research, 30, 667-672.

Melin, A. D., Kline, D. W., Hickey, C. M., \& Fedigan, L. M. (2013). Food search through the eyes of a monkey: A functional substitution approach for assessing the ecology of primate color vision. Vision Research, 86, 87-96.

Nickla, D. L., \& Wallman, J. (2010). The multifunctional choroid. Progress in Retinal and Eye Research, 29, 144-168.

Wells, D. L., McDonald, C. L., \& Ringland, J. E. (2008). Color preferences in gorillas (Gorilla gorilla gorilla) and chimpanzees (Pan troglodytes). Journal of Comparative Psychology, 122, 213-219. 